\title{
Vacinação antipneumocócica em adultos com doença invasiva pneumocócica: análise num concelho do Norte de Portugal
}

Tomás Silva, ${ }^{1}$ Luís Guedes, ${ }^{1}$ Diogo Queiroz Almeida, ${ }^{1}$ Filipa Malcata, ${ }^{2}$ Gustavo Duarte, ${ }^{1}$ Jaime Baptista ${ }^{1}$

\section{RESUMO}

Objetivos: Descrever os casos de doença invasiva pneumocócica notificados em adultos residentes no concelho de Matosinhos entre 2016 e 2018 e avaliar o seu estado vacinal, de acordo com o preconizado na Norma de Orientação Clínica n 011/2015, da Direção-Geral da Saúde, que estabelece os grupos de risco em que a vacinação antipneumocócica de adultos é recomendada em Portugal.

Métodos: Análise retrospetiva dos casos confirmados de doença invasiva pneumocócica em adultos (idade $\geq 18$ anos) residentes no concelho de Matosinhos, notificados através do Sistema Nacional de Vigilância Epidemiológica (SINAVE), entre 2016 e 2018. Análise descritiva dos casos e avaliação do estado vacinal de acordo com a Norma de Orientação Clínica ${ }^{\circ} 011 / 2015$. Resultados: Observaram-se 47 casos confirmados, 66,0\% com idade igual ou superior a 65 anos e 83,0\% com idade igual ou superior a 50 anos. Do total, 37 casos $(78,7 \%)$ apresentavam pelo menos uma das condições de risco para a doença enumeradas na norma. Destes, cinco (13,5\%) tinham registo de vacinação antipneumocócica, sendo que um $(2,7 \%)$ apresentava o esquema recomendado completo.

Conclusões: Verificou-se uma baixa proporção de casos de doença invasiva pneumocócica com vacinação antipneumocócica prévia. Vários motivos podem justificar os resultados encontrados, sendo necessária mais investigação neste âmbito. Destaca-se a importância da idade como fator de risco, justificando a discussão da sua inclusão nas recomendações. Este estudo evidencia a importância da doença invasiva pneumocócica, assim como a necessidade de avaliar a adesão à Norma n 11/2015, promover a vacinação antipneumocócica junto dos profissionais de saúde e da população e reavaliação dos critérios de vacinação.

Palavras-chave: Infeções pneumocócicas; Vacinas pneumocócicas; Adultos; Vigilância epidemiológica em saúde pública; Portugal.

\section{INTRODUÇÃO}

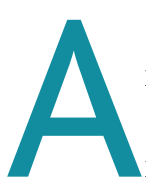

doença invasiva pneumocócica (DIP) é definida como o isolamento ou deteção de Streptococcus pneumoniae a partir de um local normalmente estéril. ${ }^{1}$ Pode manifestar-se como meningite ou como bacteriemia. ${ }^{2-3}$ Esta última pode estar associada a pneumonia, meningite ou infeção noutro local..$^{2-3}$ É mais frequente em crianças com menos de dois anos e em indivíduos com mais de 65 anos, assim como em indivíduos com imunodeficiência (inata ou adquirida), com implantes cocleares ou com patolo-

1. Unidade de Saúde Pública, ULS de Matosinhos.

2. Unidade de Saúde Pública, ACeS Porto Ocidental. gias como diabetes ou doenças respiratórias, cardíacas e hepáticas crónicas. ${ }^{2,4-5}$

Em 2017 foram notificados 6,2 casos confirmados de DIP por 100.000 habitantes na União Europeia, ${ }^{6}$ embora exista uma grande variabilidade entre os diversos países. ${ }^{6}$ Em Portugal, a DIP foi definida como doença de notificação obrigatória em $2014,{ }^{7}$ tendo sido notificados 2,9 casos confirmados por 100.000 habitantes em $2017 .^{6}$

A DIP tem uma elevada carga de doença associada. ${ }^{2,8-9}$ Destaca-se a letalidade que apresenta nos países desenvolvidos, que se estima entre 11 a $30 \%$ nos adultos com a doença, ${ }^{8}$ assim como os elevados custos associados à mesma. ${ }^{2}$ 
Na prevenção da DIP são utilizados dois tipos de vacinas: a vacina polissacárida e a vacina conjugada. ${ }^{10} \mathrm{~A}$ vacina polissacárida, a primeira a surgir no mercado, contém antigénios capsulares polissacarídeos do Streptococcus pneumoniae e confere proteção contra 23 serótipos da bactéria, com uma eficácia entre $56 \%$ a $75 \%$ nos serótipos abrangidos. ${ }^{10} \mathrm{~A}$ vacina conjugada contém uma proteína imunogénica conjugada e abrange atualmente 13 serótipos da bactéria (inicialmente apenas contra sete serótipos)..$^{10}$ Eficaz contra as formas invasivas da doença e aprovada para uso em crianças, o surgimento da vacina conjugada em 2000 levou a uma redução na incidência de DIP causada pelos serótipos abrangidos pela vacina em menores de cinco anos, mas também em idosos. ${ }^{10}$

A vacina conjugada foi aprovada para uso em adultos no final de 2011. ${ }^{10}$ Nos Estados Unidos é recomendada a todos os indivíduos a partir dos 65 anos, assim como a indivíduos adultos com menos de 65 anos com fatores de risco para a doença. ${ }^{10}$ Numa revisão, abrangendo 31 países europeus, foram encontradas orientações para a vacinação antipneumocócica de adultos em 28. ${ }^{11}$ Nesses, a vacinação é recomendada em adultos com condições de risco, embora a lista de condições abrangidas e o esquema de vacinação sejam variáveis. ${ }^{11}$ Um total de 24 países possuem recomendações baseadas unicamente na idade, independentemente das condições de risco, com idades de início de vacinação variáveis entre os 50 e os 65 anos. ${ }^{11}$

Em Portugal, a vacina conjugada começou a ser comercializada em 2001 e foi introduzida no Programa Nacional de Vacinação em 2015. ${ }^{12}$ No que diz respeito à vacinação de adultos, em 2015 foi publicada a Norma de Orientação Clínica no 011/2015, da Direção-Geral da Saúde, que define os grupos de risco em que a vacinação é recomendada, assim como um grupo mais restrito de condições em que a vacina conjugada é gratuita: presença de fístulas de líquido cefalorraquidiano, implantes cocleares ou algumas situações de imunodepressão grave. ${ }^{13} \mathrm{O}$ esquema recomendado prevê a vacinação inicial com a vacina conjugada, seguida da vacina polissacárida seis a doze meses depois. ${ }^{13}$ Nos doentes imunodeprimidos, doentes com fístulas de líquidocefalorraquidiano e nos portadores de implantes cocleares é recomendada a administração da vacina polissacárida a cada cinco anos. ${ }^{13}$
Apesar de não existirem recomendações oficiais para a vacinação de adultos sem condições de risco, a vacinação de adultos a partir dos 65 anos em Portugal é recomendada pelo Grupo de Doenças Respiratórias da Associação Portuguesa de Medicina Geral e Familiar, ${ }^{14}$ sendo também proposta pela Fundação Portuguesa do Pulmão. ${ }^{15}$

O presente trabalho pretende descrever os casos de DIP notificados em adultos residentes do concelho de Matosinhos entre 2016 e 2018 (i.e., a partir da introdução da Norma de Orientação Clínica no 011/2015, da Direção-Geral da Saúde) e caracterizar o seu estado vacinal de acordo com o preconizado na referida norma.

\section{MÉTODOS}

Análise retrospetiva de todos os casos confirmados de DIP em indivíduos com idade igual ou superior a 18 anos residentes no concelho de Matosinhos, notificados através do Sistema Nacional de Vigilância Epidemiológica (SINAVE) entre 2016 e 2018. Todos os dados foram recolhidos no âmbito da vigilância epidemiológica da DIP, nomeadamente através dos inquéritos epidemiológicos realizados, tendo como fontes de informação a notificação preenchida pelo médico notificador, a consulta do processo clínico do doente, o contacto telefónico com médico notificador e, sempre que possível, o contacto telefónico ou presencial com o doente. Para todos os casos foram recolhidas as seguintes variáveis: idade no momento do diagnóstico, sexo, estado prévio de vacinação antipneumocócica, apresentação clínica da doença, duração do internamento, necessidade de admissão em unidade de cuidados intensivos e informação relativa à evolução clínica (nomeadamente sobrevivência e existência de sequelas da doença). Foi ainda avaliada a presença de todas as condições de risco para DIP listadas na Norma de Orientação Clínica no 011/2015, da Direção-Geral da Saúde, definindo-se variáveis relativas à recomendação para vacinação antipneumocócica e à sua gratuitidade.

Foi realizada uma análise descritiva de todos os casos de forma agregada. Foi avaliada a existência de vacinação antipneumocócica prévia de acordo com a recomendação para a vacinação, tendo sido descrita a evolução da doença nos indivíduos vacinados e não vacinados. 
As variáveis categóricas são apresentadas através das suas frequências absolutas e relativas. As variáveis contínuas são descritas como média \pm desvio-padrão, após confirmação da sua distribuição normal, através dos valores de assimetria e curtose da sua distribuição e dos testes Kolmogorov-Smirnove Shapiro-Wilk. Toda a análise foi realizada com recurso ao software IBM Statistical Package for Social Sciences ${ }^{\circledR}$, v. 24.0.

O presente estudo foi aprovado pela Direção Executiva do Agrupamento de Centros de Saúde da Unidade Local de Saúde (ULS) de Matosinhos e pelo Conselho de Administração da ULS de Matosinhos. O protocolo de estudo foi submetido à Comissão de Ética da ULS de Matosinhos que deliberou, por unanimidade, nada opor à sua realização (Parecer no 44/CE/JAS).

\section{RESULTADOS}

Entre 2016 e 2018 foram notificados 47 casos confirmados de DIP em adultos residentes no concelho de Matosinhos: oito em 2016, 14 em 2017 e 25 em 2018. No momento do diagnóstico, os doentes apresentavam idades compreendidas entre 26 e 91 anos (idade média $67,9 \pm 15,2$ anos), com 31 doentes $(66,0 \%)$ com idade igual ou superior a 65 anos e 39 (83,0\%) com idade igual ou superior a 50 anos. Do total de casos, $30(63,8 \%)$ eram do sexo masculino.

À admissão, 44 doentes (93,7\%) apresentavam bacteriemia, sendo a apresentação mais frequente pneumonia com bacteriemia (39 casos; 83,0\%). Em quatro casos $(8,5 \%)$ a apresentação foi de meningite, um dos quais $(2,1 \%)$ com bacteriemia associada, enquanto quatro doentes $(8,5 \%)$ manifestaram bacteriemia com origem em foco nem respiratório nem meníngeo. Em nenhum dos casos foi pesquisado o serótipo responsável pela doença.

Observou-se um total de seis óbitos (12,8\%). Entre os 41 casos não fatais, todos necessitaram de internamento, sendo que $10(24,4 \%)$ foram admitidos em unidade de cuidados intensivos. A duração média de internamento foi de 17,6 $\pm 11,3$ dias. Verificou-se a existência de sequelas da doença em seis casos $(14,6 \%)$ não fatais.

Previamente à admissão, 37 doentes $(78,7 \%)$ apresentavam pelo menos uma das condições de risco para a DIP elencadas na Norma de Orientação Clínica ${ }^{\circ}$ 011/2015 e, consequentemente, com recomendação para vacinação antipneumocócica. A diabetes mellitus com necessidade de terapêutica farmacológica $(n=15$, $31,9 \%)$, a doença respiratória crónica ( $n=14,29,8 \%)$, a doença cardíaca crónica $(n=14,29,8 \%)$ e a doença neoplásica ativa $(n=8,17,0 \%)$ foram as condições mais frequentemente registadas. De salientar que sete casos $(14,9 \%)$ tinham direito à vacina conjugada de forma gratuita.

Entre os doentes com mais de 65 anos, 28 (90,3\%) apresentavam pelo menos uma das condições de risco presentes na norma $n^{\circ} 011 / 2015$.

A Tabela I apresenta o estado vacinal dos casos de DIP prévio à admissão, apresentado de acordo com a recomendação para a vacinação.

Relativamente ao estado vacinal, cinco doentes $(10,6 \%)$ apresentavam registo de vacinação antipneumocócica prévia, correspondendo a $13,5 \%$ dos doentes com recomendação vacinal. Destes, um $(2,1 \%)$ apresentava o esquema recomendado completo (vacina conjugada de 13 valências seguida de vacina de 23 valências). Os cinco doentes vacinados apresentavam indicação para vacinação de acordo com a Norma $n^{\circ}$ 011/2015 (um deles com acesso gratuito à vacina conjugada). Entre os 42 doentes não vacinados, 32 (76,2\%) tinham indicação para vacinação, quatro dos quais com direito a vacina conjugada de forma gratuita $(9,5 \%)$.

Os seis óbitos observados ocorreram em indivíduos não vacinados com indicação para vacinação (um deles com direito a vacina conjugada gratuita). Nenhum dos 10 doentes internados em unidade de cuidados intensivos apresentava registo vacinal, sendo que sete (70,0\%) tinham indicação formal para vacinação antipneumocócica. Entre os seis indivíduos que ficaram com sequelas da doença, cinco $(83,3 \%)$ tinham indicação para vacinação. Desses, apenas um (20,0\%) tinha registo de administração de vacina pneumocócica conjugada de 13 valências.

\section{DISCUSSÃO}

No presente estudo observou-se um aumento anual no número de casos notificados de DIP em adultos residentes no concelho de Matosinhos. Este aumento pode estar relacionado, pelo menos parcialmente, com uma maior notificação por parte dos clínicos, uma vez que no concelho de Matosinhos o número total de notificações de doenças de notificação obrigatória 
TABELA I. Estado vacinal dos casos de doença invasiva pneumocócica prévio à admissão, de acordo com a recomendação para a vacinação pela Norma de Orientação Clínica n 011/2015

\begin{tabular}{|c|c|c|c|c|}
\hline & & \multicolumn{3}{|c|}{ Recomendação para vacinação } \\
\hline & & Vacinação recomendada & Vacinação não recomendada & Total \\
\hline \multirow[t]{3}{*}{ Estado Vacinal Prévio } & Vacinado & $5(10,6 \%)$ & $0(0 \%)$ & $5(10,6 \%)$ \\
\hline & Não Vacinado & $32(68,1 \%)$ & $10(21,3 \%)$ & $42(89,4 \%)$ \\
\hline & Total & $37(78,7 \%)$ & $10(21,3 \%)$ & $47(100 \%)$ \\
\hline
\end{tabular}

também sofreu um aumento anual no período em estudo: 185 em 2016, 224 em 2017 e 276 em 2018. ${ }^{16}$ Uma vez que não se encontra estudada a subnotificação das doenças de notificação obrigatória ou da DIP em Portugal, esta tendência evolutiva deve ser interpretada com precaução. Consequentemente, o número total de casos no período em estudo e a magnitude da DIP em Matosinhos pode ser superior ao notificado, o que reforça a importância da doença e da sua notificação no âmbito da vigilância epidemiológica, assim como do papel das Unidades de Saúde Pública para alertarem para a importância da correta notificação.

A análise descritiva dos casos de Matosinhos vai ao encontro da epidemiologia da doença descrita na literatura: ligeiro predomínio no sexo masculino, ${ }^{4,6}$ maior incidência em idades avançadas ${ }^{6,10} \mathrm{e}$ associada à presença de condições de risco para a doença ${ }^{4-5}$ De referir que apenas foram analisadas as condições clínicas listadas na Norma de Orientação Clínica no 011/2015, não tendo sido avaliados outros fatores de risco importantes para a doença, como o tabagismo e o alcoolismo, ${ }^{4-5}$ que poderiam também estar presentes, em particular nos casos sem recomendação vacinal.

É também evidente nesta análise o impacto da DIP, patente na letalidade observada $(12,8 \%)$, na duração média de internamento (17,6 $\pm 11,3$ dias), na proporção de doentes com necessidade de internamento em unidade de cuidados intensivos $(24,4 \%)$ e com sequelas da doença $(14,6 \%)$, com repercussões económicas e sociais. Estes dados reforçam a importância da prevenção da mesma.

Considerando que existe uma vacina eficaz disponível, recomendada inclusivamente em norma de orientação clínica, seria desejável e expectável a sua ampla utilização. Contudo, nesta análise verificou-se que, dos
37 casos de DIP em adultos residentes no concelho de Matosinhos com recomendação para a vacina, apenas cinco doentes (13,5\%) tinham registo de vacinação antipneumocócica. Salientando que os resultados são referentes a uma população restrita, a baixa proporção de casos de DIP com vacinação antipneumocócica prévia demonstra a necessidade de avaliar a adesão à norma $n^{\circ} 011 / 2015$, reforçando também a importância e urgência da promoção da vacinação antipneumocócica.

Adicionalmente, dos cinco doentes vacinados, apenas um possuía o esquema recomendado completo. A indisponibilidade da vacina polissacárida de 23 valências, anunciada em 2017, ${ }^{17}$ poderá ter sido um fator crítico. A reintrodução da sua comercialização em 2019 perfila-se como uma oportunidade para relembrar a vacinação antipneumocócica, assim como para completar o esquema nos doentes que já receberam a vacina conjugada de 13 valências.

No presente estudo não foram avaliados os motivos para a não vacinação. Assim, é impossível afirmar se a vacinação não foi prescrita, seja por ausência de contacto dos doentes com os serviços de saúde ou incumprimento da norma por parte dos clínicos, ou se a vacina foi aconselhada pelos clínicos mas não existiu adesão pelos doentes, nomeadamente por não reconhecerem a importância da vacina ou por barreiras económicas ao seu acesso. Perante esta situação, é prioritário estudar os motivos para a não vacinação, assim como o custo benefício do alargamento do grupo de doentes para os quais a vacinação é gratuita. Não obstante, a importância da vacinação deve ser promovida simultaneamente junto dos profissionais de saúde e da população geral, em particular nos indivíduos pertencentes aos grupos de risco para a doença, onde a não vacinação deve ser encarada como uma oportunidade 
de educação para a saúde, contribuindo para a capacitação dos indivíduos e melhoria do nível de saúde da população. ${ }^{18}$

A estratégia de vacinação de adultos deve também ser discutida. A norma no 011/2015 recomenda a vacinação antipneumocócica na presença de certas condições de risco, incluindo algumas doenças crónicas e diversas formas de imunodepressão, de forma análoga ao verificado em recomendações internacionais. ${ }^{11}$ Contudo, em oposição a diversos países europeus em que existem recomendações contendo a idade como critério (com idades a partir das quais a vacinação é recomendada a variar entre os 50 e 65 anos), ${ }^{11}$ a norma portuguesa não contempla esta indicação. Neste estudo, $66,0 \%$ dos doentes com DIP apresentavam idade igual ou superior a 65 anos e $83,0 \%$ idade igual ou superior a 50 anos. Verificou-se ainda que 28 dos 31 doentes com mais de 65 anos $(90,3 \%)$ apresentavam, pelo menos, uma das condições de risco presentes na norma $\mathrm{n}^{\circ}$ $011 / 2015$. Esta sobreposição advoga a favor da existência de uma recomendação pela idade, na medida em que não só a incidência da doença é superior em idades avançadas, ${ }^{10}$ como a população idosa tende a apresentar simultaneamente outros fatores de risco para a doença. ${ }^{5}$ Estes dados vão ao encontro das recomendações do Grupo de Doenças Respiratórias da Associação Portuguesa de Medicina Geral e Familiar ${ }^{14}$ e da Fundação Portuguesa do Pulmão, ${ }^{15}$ que aconselham a vacinação antipneumocócica acima dos 65 anos e reforçam a necessidade de reavaliar as recomendações da Direção-Geral da Saúde para a vacinação antipneumocócica.

Em nenhum dos casos foi pesquisado o serótipo responsável pela doença. Assim, não é possível avaliar o número de casos causados pelos serótipos incluídos nas vacinas disponíveis e, consequentemente, a proporção de casos potencialmente evitáveis pela vacinação. Este facto realça a importância de um sistema de vigilância epidemiológico robusto, conciliando dados clínicos, epidemiológicos e serológicos.

Este estudo evidencia a importância da DIP, assim como a necessidade de avaliar a adesão à norma $\mathrm{n}^{\circ}$ 011/2015 e de promover a vacinação antipneumocócica junto dos profissionais de saúde e da população. Neste âmbito considera-se oportuna uma reavaliação dos critérios para a vacinação.
Por fim, o investimento na recolha de dados serológicos é essencial para aumentar a robustez da vigilância epidemiológica da DIP, permitindo não só uma melhor monitorização da evolução da doença, como do impacto da vacinação.

\section{REFERÊNCIAS BIBLIOGRÁFICAS}

1. Despacho n $15.385-A / 2016$, de 21 de dezembro. Diário da República. $2^{\text {a }}$ Série(243).

2. Zhang D, Petigara T, Yang X. Clinical and economic burden of pneumococcal disease in US adults aged 19-64 years with chronic or immunocompromising diseases: an observational database study. BMC Infect Dis. 2018;18(1):436.

3. Ludwig E, Bonanni P, Rohde G, Sayiner A, Torres A. The remaining challenges of pneumococcal disease in adults. Eur Respir Rev. 2012;21(123): 57-65.

4. Schoenmakers MC, Hament JM, Fleer A, Aerts PC, Van Dijk H, Kimpen $J \mathrm{~L}$, et al. Risk factors for invasive pneumococcal disease. Rev Med Microbiol. 2002;13(1):29-36.

5. Curcio $D$, Cané $A$, Isturiz R. Redefining risk categories for pneumococcal disease in adults: critical analysis of the evidence. Int J Infect Dis. 2015;37:30-5.

6. European Centre for Disease Prevention and Control. Invasive pneumococcal disease: annual epidemiological report for 2017. Stockholm: ECDC; 2019.

7. Despacho n 5.855/2014, de 5 de maio. Diário da República. 2a Série(85).

8. Drijkoningen JJ, Rohde GG. Pneumococcal infection in adults: burden of disease. Clin Microbiol Infect. 2014;20 Suppl 5:45-51.

9. Van Lier A, McDonald SA, Bouwknegt M, Kretzschmar ME, Havelaar AH, Mangen MJ, et al. Disease burden of 32 infectious diseases in the Netherlands, 2007-2011. PLoS One. 2016;11(4):e0153106.

10. Gierke R, McGee L, Beall B, Pilishivili T. Pneumococal. In: Centers for Disease Control and Prevention, editor. Manual for the surveillance of vaccine-preventable diseases. Atlanta, GA: CDC; 2017. chapter 11.

11. Bonnave C, Mertens D, Peetermans W, Cobbaert K, Ghesquiere B, Deschodt $M$, et al. Adult vaccination for pneumococcal disease: a comparison of the national guidelines in Europe. Eur J Clin Microbiol Infect Dis. 2019;38(4):785-91.

12. Ferreira M, Oliveira $H$, Silva NC, Januário L, Rodrigues F. Doença invasiva pneumocócica pediátrica antes da vacinação universal: 1995 2015 [Paediatric invasive pneumococcal disease before universal vaccination: 1995 - 2015]. Acta Med Port. 2017;30(6):457-62. Portuguese

13. Direção-Geral da Saúde. Vacinação contra infeções por Streptococcus pneumoniae de grupos com risco acrescido para doença invasiva pneumocócica (DIP) - adultos ( $\geq 18$ anos de idade): norma $\mathrm{n}^{\circ}$ 011/2015, de 23/06/2015, atualizada a 06/11/2015. Lisboa: DGS; 2015.

14. Costa RP, Gonçalves C, Sousa JC. A doença pneumocócica e recomendações GRESP para a vacinação antipneumocócica na população adulta ( $\geq 18$ anos) [GRESP recommendations for anti-pneumococcal vaccination in adults]. Rev Port Med Geral Fam. 2016;32(1):70-4. Portuguese 
15. Observatório Nacional das Doenças Respiratórias. $13^{\circ}$ Relatório do Observatório Nacional das Doenças Respiratórias [Internet]. Lisboa: ONDR; 2018. Available from: https://www.ondr.pt/files/Relatorio_ ONDR_2018.pdf

16. Silva T, Baptista J. Relatório de avaliação da vigilância epidemiológica das doenças de notificação obrigatória - ano 2018. Matosinhos: Unidade Local de Saúde de Matosinhos; 2019.

17. Direção-Geral da Saúde. Vacinação contra infeções por Streptococcus pneumoniae de grupos com risco acrescido para doença invasiva pneumocócica (DIP): orientação n 017/2017, de 20/09/2017. Lisboa: DGS; 2017.

18. Santos P, Hespanhol A. Recusa vacinal: o ponto de vista ético [An ethical view of vaccine refusal]. Rev Port Med Geral Fam. 2013;29(5):32833. Portuguese

\section{CONFLITO DE INTERESSES}

Os autores declaram não ter quaisquer conflitos de interesse.

\section{FONTE DE FINANCIAMENTO}

O trabalho relatado neste manuscrito não foi objeto de qualquer tipo de financiamento externo (incluindo bolsas e investigação).

\section{APROVAÇÃO ÉTICA}

Os autores declaram que os procedimentos seguidos estavam de acordo com o protocolo de estudo submetido à Comissão de Ética da Unidade Local de Saúde de Matosinhos, que deliberou, por unanimidade, nada opor à sua realização (Parecer n 44/CE/JAS).

\section{ENDEREÇO PARA CORRESPONDÊNCIA \\ Tomás Silva \\ E-mail: tomas.silva@ulsm.min-saude.pt \\ https://orcid.org/0000-0002-8087-5469}

Recebido em 27-08-2019

Aceite para publicação em 03-12-2019

\section{ABSTRACT \\ PNEUMOCOCCAL VACCINATION IN ADULTS WITH INVASIVE PNEUMOCOCCAL DISEASE: AN OVERVIEW IN A PORTUGUESE COMMUNITY}

Objectives: To describe all cases of invasive pneumococcal disease reported in adult residents of Matosinhos between 2016 and 2018 while assessing their vaccination status and compliance with clinical guideline no. 011/2015, which establishes the risk groups to whom pneumococcal vaccination of adults is recommended in Portugal.

Methods: Retrospective analysis of the confirmed cases of invasive pneumococcal disease in adults (aged $\geq 18$ years) residing in the municipality of Matosinhos, notified through the Sistema Nacional de Vigilância Epidemiológica (SINAVE) between 2016 and 2018. Descriptive analysis of the cases and evaluation of the vaccination status according to the clinical guideline no. 011/2015.

Results: There were 47 confirmed cases, $66.0 \%$ in patients aged 65 years and older, and $83.0 \%$ aged 50 years or older. In all cases, $37(78.7 \%)$ had at least one of the risk conditions for the disease listed in the guideline. Of these, five (13.5\%) had pneumococcal vaccination records, and one (2.7\%) had the complete recommended regimen.

Conclusions: A reduced proportion of invasive pneumococcal disease cases was previously vaccinated. Several reasons may justify the results found and more research is needed. We emphasize the importance of age as a risk factor, justifying the evaluation of its inclusion in the recommendations. This study highlights the importance of invasive pneumococcal disease, as well as the need to evaluate the compliance with Portuguese guideline no. $011 / 2015$, to promote pneumococcal vaccination among both health professionals and the general population and the need to re-evaluate the current recommendations.

Keywords: Pneumococcal infections; Pneumococcal vaccines; Adults; Public health surveillance; Portugal. 